\title{
Control System in Mechatronics -A Review
}

\author{
Shunmathi $\mathbf{M}^{*}$ \\ Thiagarajar college of Engineering
}

*Corresponding Author: Shunmathi M, Thiagarajar college of Engineering

\begin{abstract}
This paper discussing about existing and upcoming control model of the system.In various types of contolling methods like electronic control model of (PID) Proportional Integral and Derivative control, adaptive control,crucise control based upon servo mechanism.Generally mechatronics is synergistic integration of mechanical engineering with electronics and intelligent computer control design of manufacture of product and material handling and process. In some of Techniques like Machine learning, Deep learning Techniques and AI.In major rule according to design and implemenation of robot and to communicate man to robot,Two way communication Transmitter and receiver and controller.
\end{abstract}

Keywords: Artificial Intelligence,Engine management system,control modelling.

\section{INTRODUCTION}

Mechatronics major rule play in controller design, plant model, Engine control, active suspension, chain mechanism. In some of the application like automatic car parking system, Temperature monitoring system. Embedded system and microcontroller, Arudino using for programming made design of robot. In categorized control system main elements of sensor, controller, actuator helpful for system design. The controller is 'mind' of the mechatronic system, which processes user commands and sensed signals to generate command signals to be sent to the actuator in the system. Actuators are devices that can convert electrical energy to mechanical energy. The user commands are obtained from a variety of devices including command buttons, graphical user interfaces (GUIs), touch screens. The combination of lab view system software NI and input and output configuration of RIO hardware helpful for robot movement.

\section{ConTrol System}

$>$ In general representation of control system is output quantity is controlled by varying the input quantity is called control system. According to two classification open loop, closed loop.

\section{Open loop:}

\section{Input}

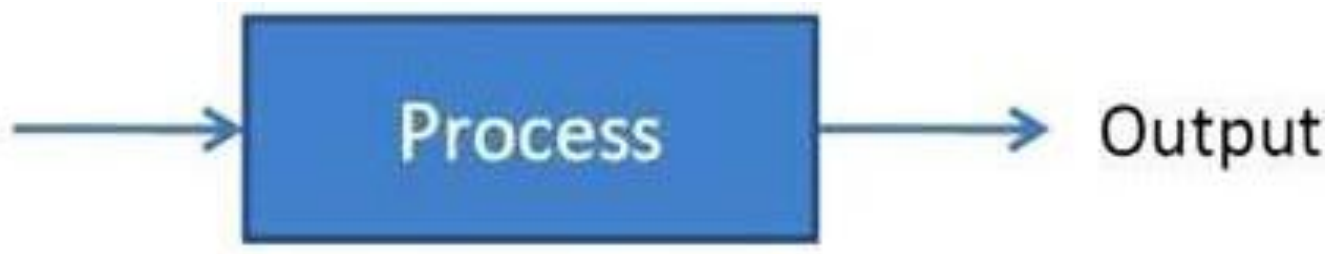

\section{Closed loop:}

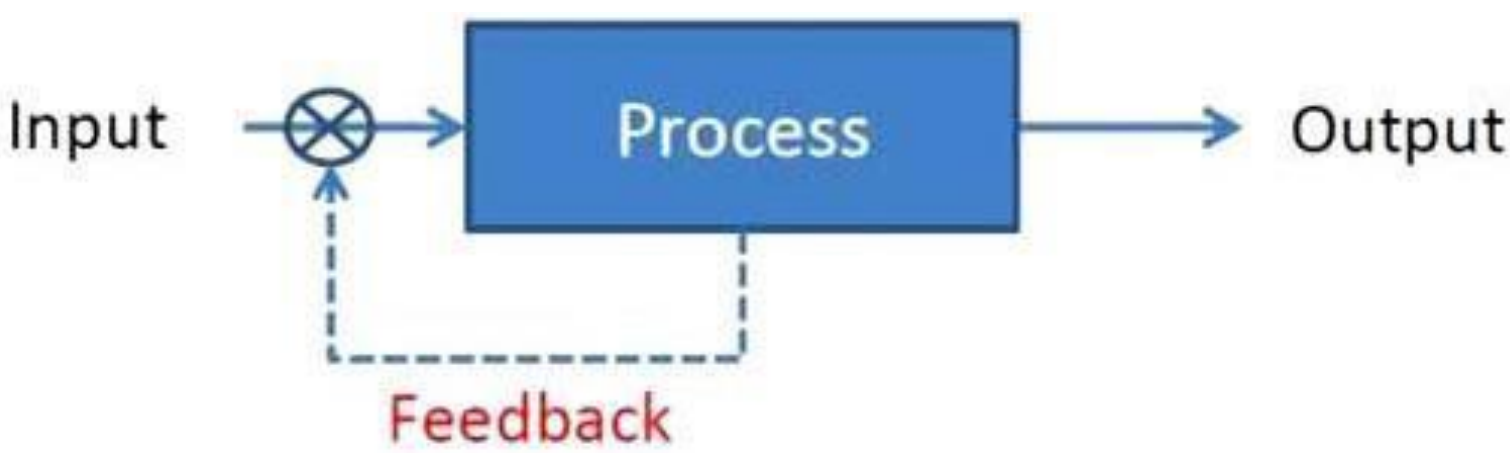


$>$ The functionalities of a control system can be automated by using a digital computer as shown in figure. Those sensors are replace the eyes, actuators are replace the muscles another one computer replace the human brain.

$>$ Every mechatronics system has some sensors to monitor and measure the status of the process control variables. The performance specifications will describe how the closed-loop system should perform and will include good regulation against disturbances, desirable responses to commands, realistic actuator signals, low sensitivities and robustness.

$>$ Desired Output/Reference Input - In systems where it is desired that the output follows the input this signal sets the desired output level. In a cruise control system this would be an electrical signal corresponding to the desired output speed.

> Plant/Process/System - refers to the system we want to control. It generally refers to a physical process which we can either model. In the example of the car cruise control system, it encompasses the car dynamics (Newton's Laws). The output signal is the speed of the car.

$>$ Transducer - The role of the transducer is to convert the output signal to an equivalent electrical system (e.g. from kinetic energy to electrical energy). This facilitates the comparison of the output signal to the input.

$>$ Error Signal - This an electrical signal that is the difference between the desired and true output signals.

$>$ Controller - This is a purposely designed system to modify the behavior of the plant.

$>$ Disturbance Input - External/environmental factors that affect plant behavior (e.g. Wind, Gradient).

\section{Control System Representation Diagram}
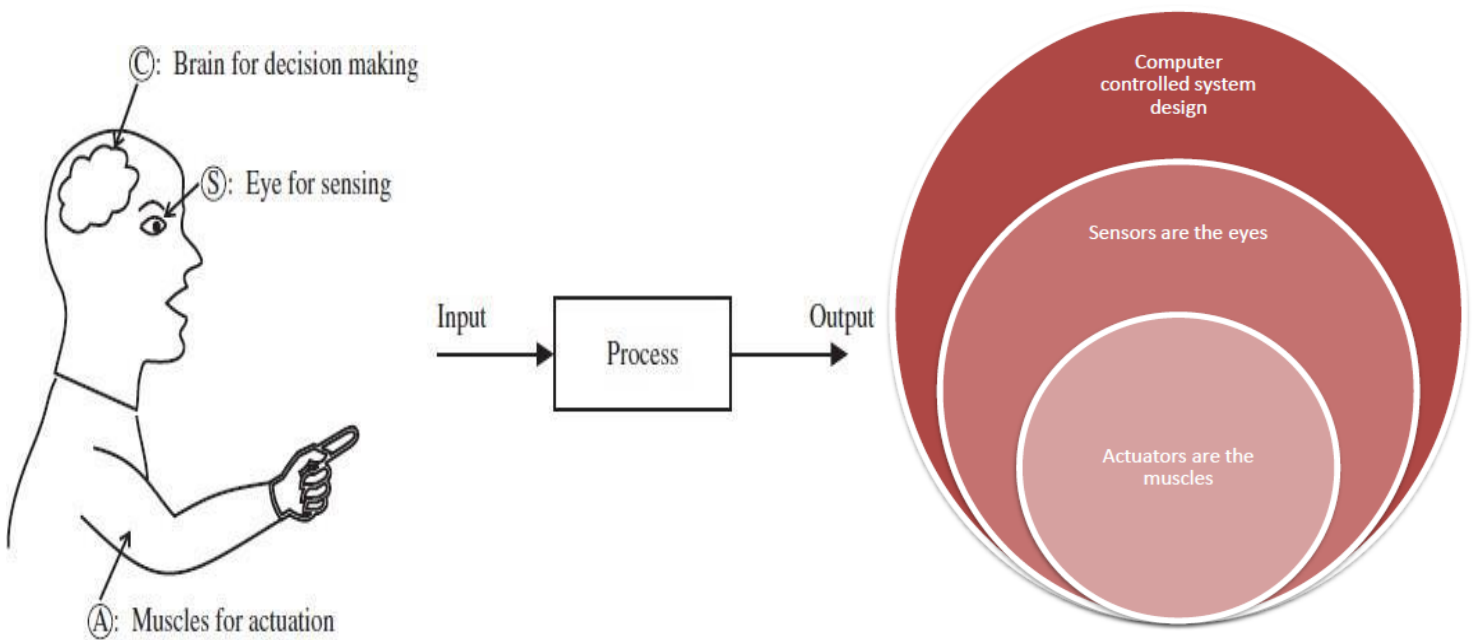

\section{Symbolic Diagram of Vehicle Speed Control}

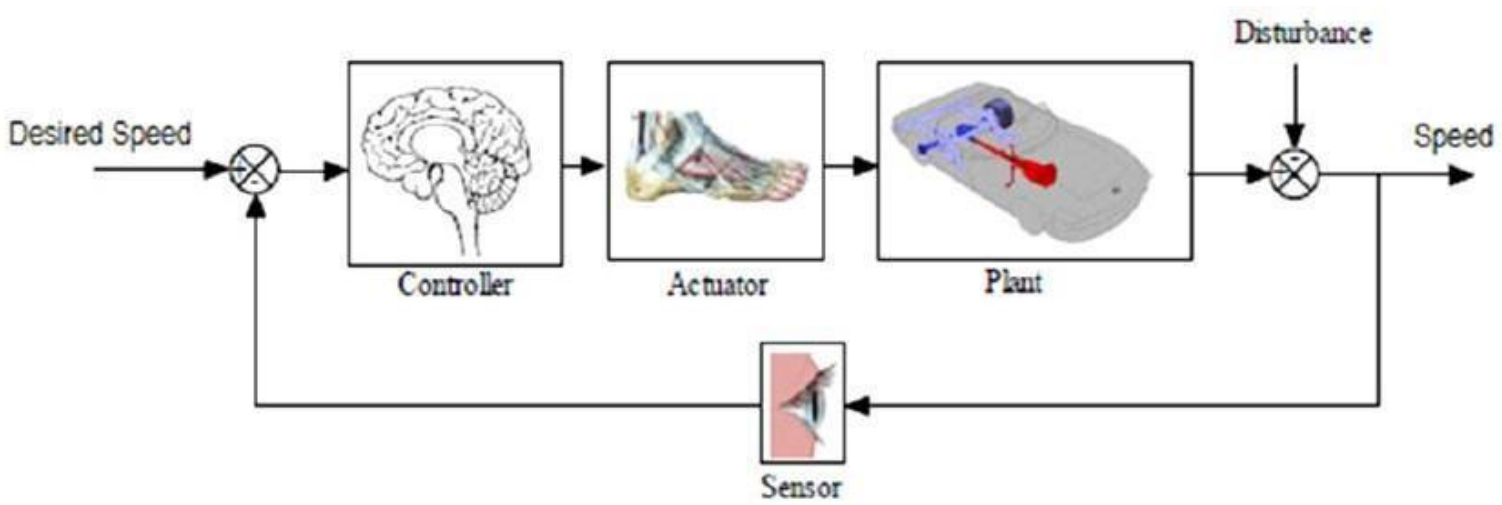

\subsection{Engine Management System}

In EMS is electronic control unit that controls the running of an engine by monitoring the engine speed performance. It also measures the exact quantity of fuel required to start a engine. 


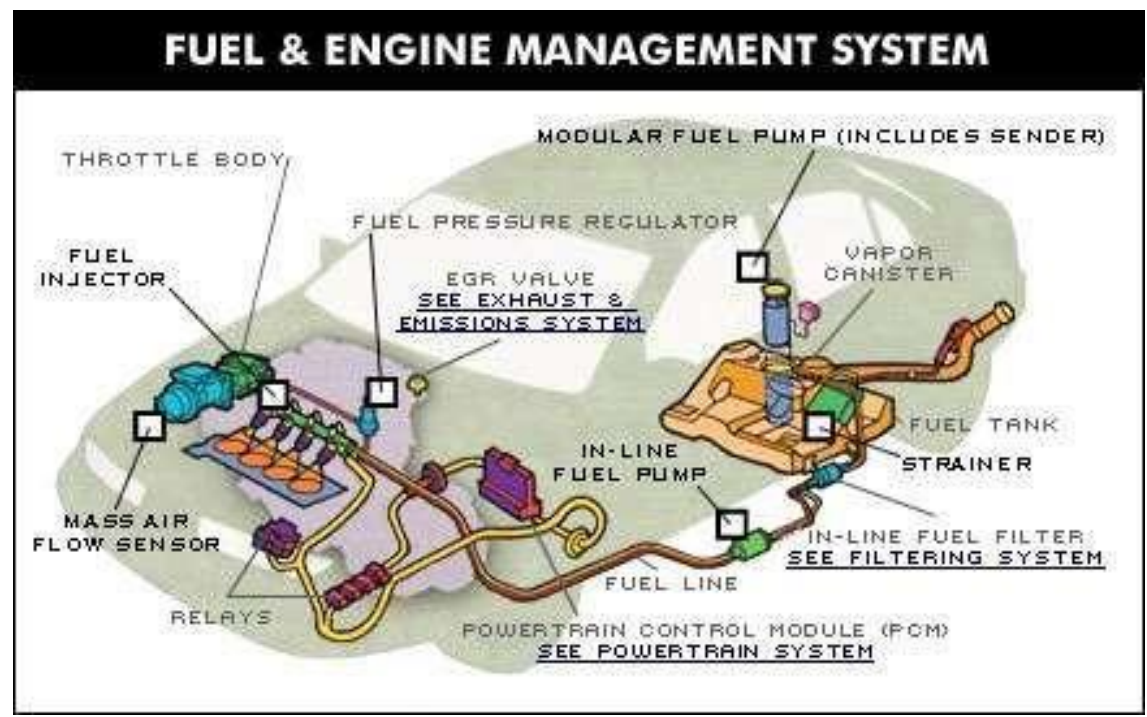

Electronic Fuel Injection System, Air Induction System/Control, Fuel Delivery System/Control, Electronic Control System, Electronic control unit, Fuel Injector sequential, Air flow sensor, Air temperature sensor, Throttle Position sensor (TPS)Temperature sensor, Oxygen sensor, Idle air control valve (IACV),MAP sensor, Knock Sensor, Engine Speed Sensor, Engine Oil Sensor, Crankshaft sensor, Camshaft Sensor and Multi-point fuel injection

\subsection{Electronic Fuel Injection System Diagram}

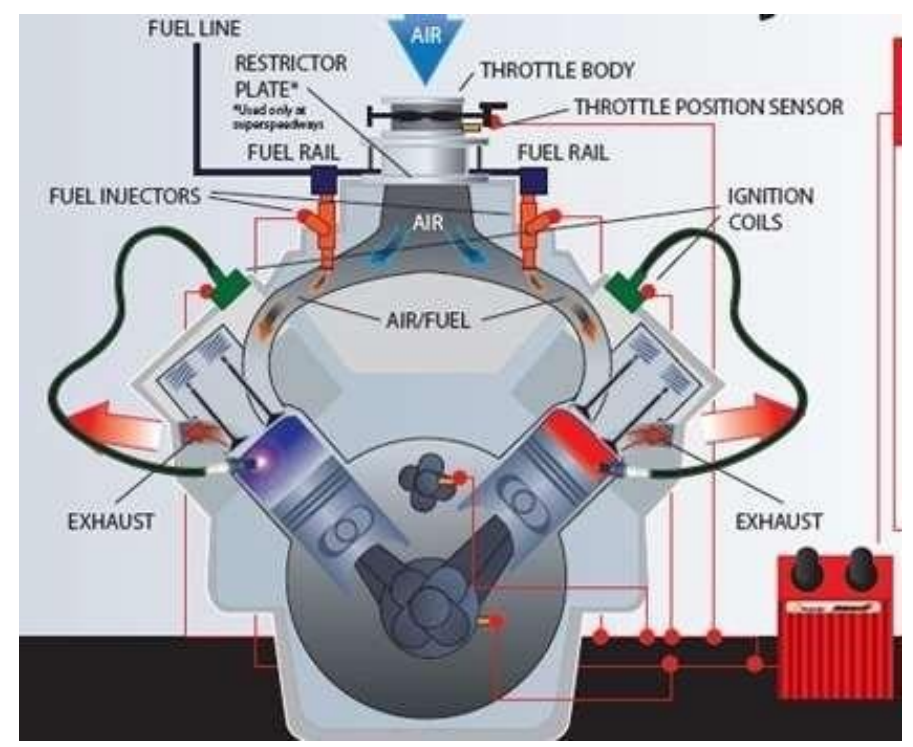

Determine the amount of fuel to inject into the engine and when to fire the spark plugs.Achieve the most horse power with the least amount of Fuel.

\subsection{Electronic Control Unit (ECU)}

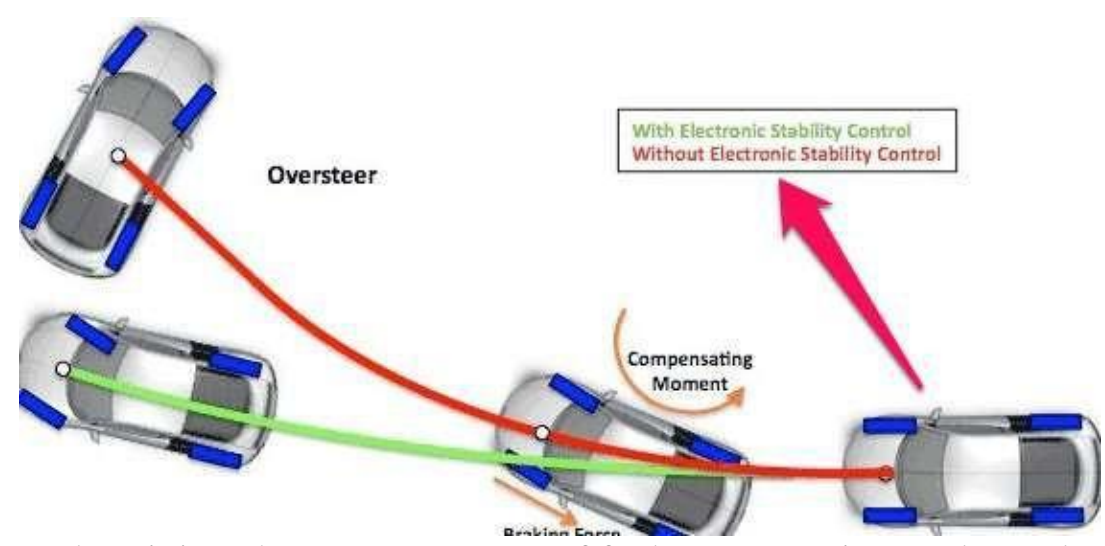

$>$ It helps to analyse inject the correct amount of fuel at correct time and speed. 

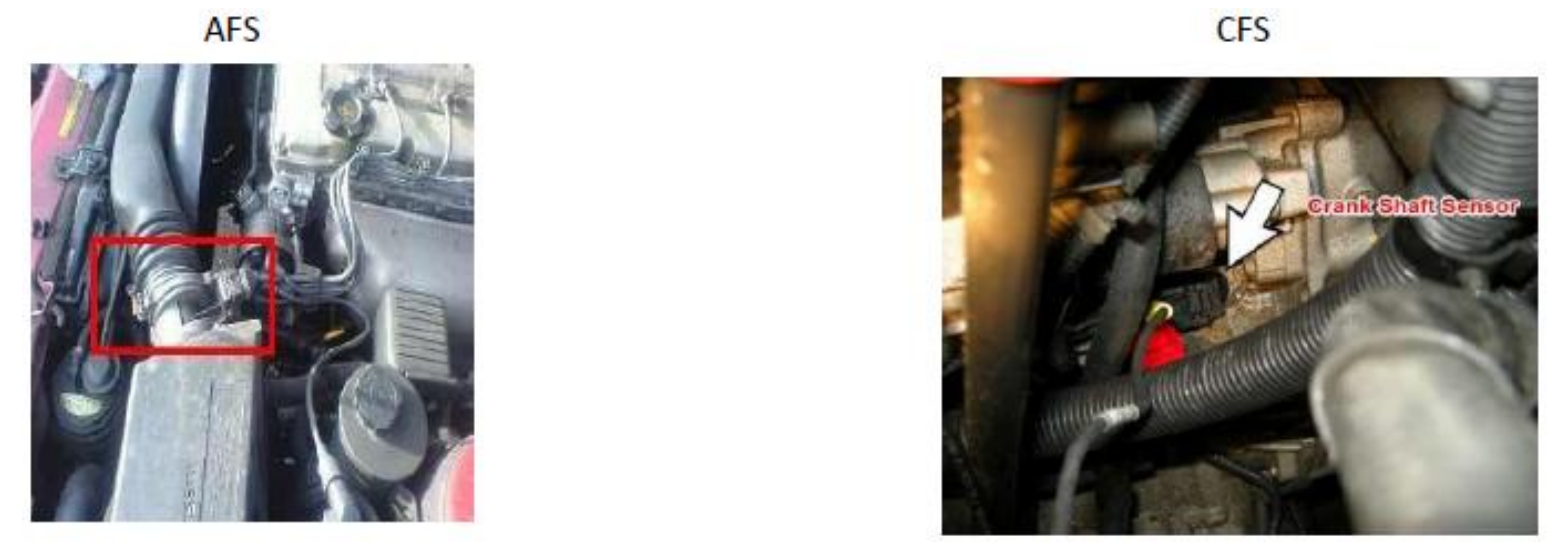

An air flow meter is a device that measures the actual speed of the air flowing through the engine in different segments.

It is used to monitor the position and rotational speed of crankshaft and this information will be used by the EMS to control the ignition system timing and other engine parameter.

\subsection{Robot Path Planning And Methods}

They are several methods to define a robot path and line follower method, Teach pendant several classification according to test, teach, repeat modes, Point to Point, continuous path, controlled path. line follower method manually define man made design robot to travel the particular distance and left, right movement turning to be automatically program itself. Robot programming is apply to microcontroller, arudino, Raspberry PI, RIO hardware to communicate robot to human.

\subsection{Mobile Robot Model}

Autonomous navigation has been applied to different types of mobile robots. This work, consider the Khepera IV mobile robot which has two independent driving wheels that are responsible for orienting and commanding the platform by acting on the speed of each wheel.

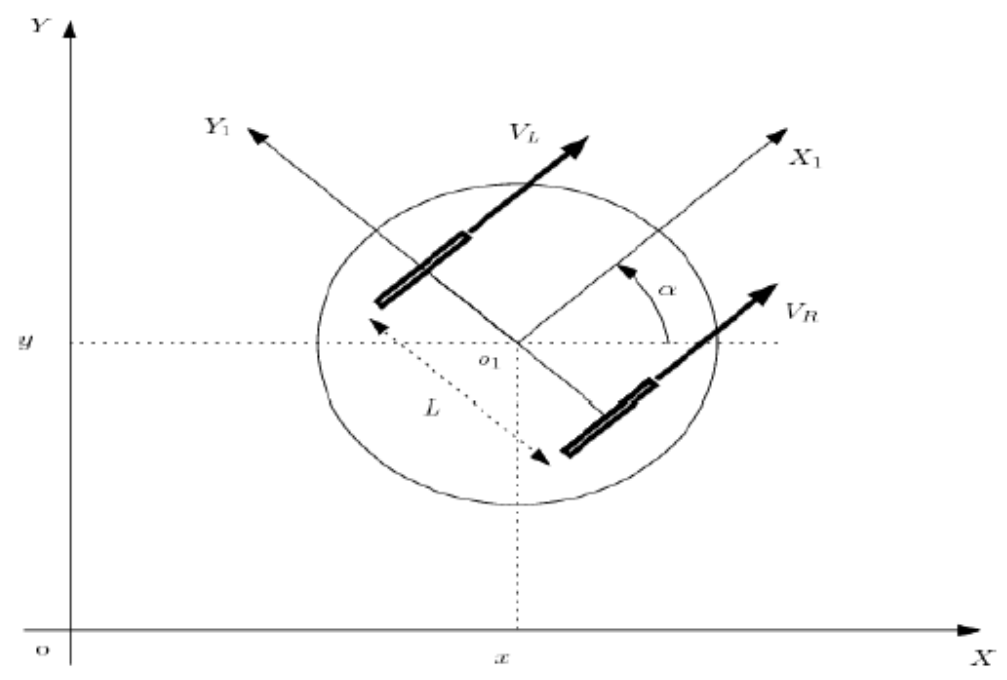

Fig1. Schematic Model of Khepera IV.

The kinematic model of a non-holonomic mobile robot is given as follows:

$\mathrm{dx} / \mathrm{dt}=\mathrm{VR}+\mathrm{VL} / 2 \cos \propto$

$\mathrm{dy} / \mathrm{dt}=\mathrm{VR}-\mathrm{VL} / 2 \sin \propto$

$\mathrm{d} \propto / \mathrm{dt}=\mathrm{VR}-\mathrm{VL} / \mathrm{L}$

where, $\mathrm{x}, \mathrm{y}=$ Cartesian coordinates

$\propto=$ angle between robot direction $\mathrm{X}$ axis

$\mathrm{L}=$ distance between the two wheels 


\section{Proposed WORK}

The proposed work performed with help of a spy robot used to monitor and keep watch of areas not easily accessible by humans. Surveillance is the process of monitoring a situation, an area or a person. It is generally practiced in a military scenario where surveillance of borderlines and enemy territory is essential to for the country's safety. Human surveillance is achieved by deploying personnel near sensitive areas in order to constantly monitor changes.

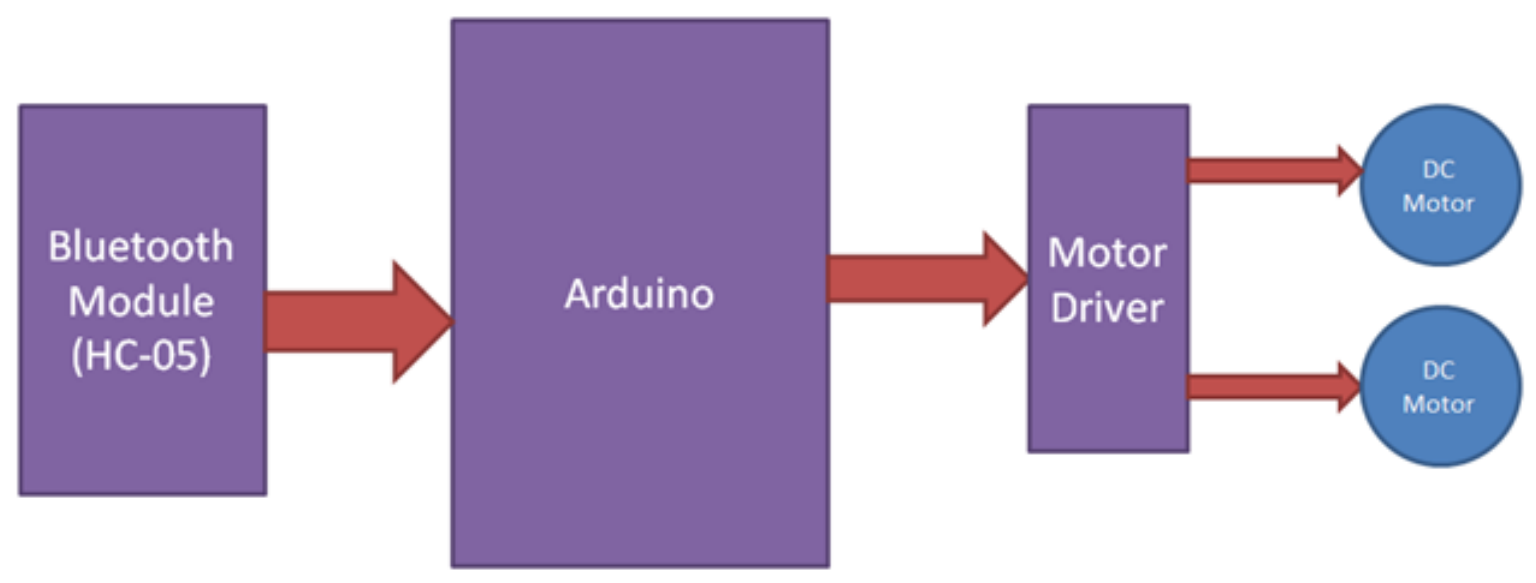

\section{Block Diagram of Arudino Controlled Robot}

To design a Robot that can be operated using Android mobile phone. The controlling of the Robot is done wirelessly through Android smart phone using the Bluetooth feature present in it. Android smart phone is used as a remote control for operating the Robot. Including Wi-Fi, Bluetooth, and wireless data over a cellular. Android provides access to a wide range of useful libraries and tools that can be used to build rich applications. In addition, Android includes a full set of tools that have been built from the ground up alongside the platform providing developers with high productivity and deep insight into their applications. Bluetooth is an open standard specification for a radio frequency (RF)based, short-range connectivity technology that promises to change the face of computing and wireless communication. The controlling device of the whole system is a Microcontroller. Bluetooth module, DC motors are interfaced to the Microcontroller. The data received by the Bluetooth module from Android smart phone is fed as input to the controller. The controller acts accordingly on the DC motors of the Robot. The robot in the project can be made to move in all the four directions using the Android phone. The direction of the robot is indicated using LED indicators of the Robot system. In achieving the task the controller is loaded with a program written using Embedded ' $\mathrm{C}$ ' language.

\subsection{Circuit Explanation}

The electronic circuit controlling the robot is built around Arduino Pro Mini. The L293D motor driver IC and HC-05 Bluetooth module are interfaced to the controller board. A pair of geared DC motors are attached at the rear wheels which are interfaced with the motor driver IC. A mobile phone whose camera is used as IP web camera is mounted on the robot body. A mobile phone is mounted on the robot for live transmission of the video recording. The phone has IP camera app installed on it which records and transmits the live recording to a remote server. The app automatically uses the mobile camera as IP camera. Another mobile phone will be used to control the robot over Bluetooth. It has a custom app installed on it which can move the robot in forward, backward, left or right direction. The development of the custom app using "Rame App" is discussed in the programming guide section. In control circuitry of the robot is powered on, it initializes the controller and starts reading data from the Bluetooth module. The control commands can be passed to the robot using a custom app running on an android phone. The app has a user interface which allows moving the robot forward, backward, turn left, turn right and stop. The user has just tap the direction buttons to transfer the commands. The commands are passed by the app to the Bluetooth module interfaced in the control circuitry in the form of single character strings. The following strings are passed to transfer the control commands. These command strings are interpreted in the Arduino sketch to control the DC motors. The robot can be moved forward, backward, left or right by implementing the following input logic at the motor driver pins. On receiving the string commands, the Arduino sketch just changes the digital output at 
the input pins of the motor driver IC to control the motion of the robot. An Android smart phone is mounted on the robot to broadcast the video and audio from the robot to the control interface (computer). An internet protocol (IP) camera is a type of digital video camera, generally employed for surveillance which can send and receive data via a computer network and internet. Any of these apps mentioned above can be used for using phone as IP camera.

\subsection{Operation}

$>$ In Mobile operated spy robot circuit which can be controlled by using mobile phone

$>$ The Mobile operated spy camera robot has been designed in such a way that it can fulfill all the needs of military, police and also for personal security.

$>$ It can capture audio and video information from the surroundings and can be sending to a remote station through transmitting signal. .

$>$ It has countless application and can be used in different environments and scenarios. For instance, at one place it can be used by bomb disposal squad, while at another instance it can be used for handling mines.

It can capture video information from the surroundings and can be sending to a remote station through transmitting signal. In PID controller used to stabilize the robot by giving suitable PWM signals to the motors. The mathematical modeling of the robot would be done and it would be transformed using Laplace transform. Using MATLAB the equation would be used in PID controller and its behavior can be observed and to analyze the system to be balanced or not.

\section{Path Planning Algorithm}

In easy way to find the path from the start to the end autonomously collecting a data and sequence of actions and decision making better way. Those sequences are decided by some algorithm based on representation of the map present. These algorithms are usually designed for certain type of environment suitable for the algorithm to function.

$>$ Dijkstra's algorithm

$>\mathrm{A}^{*}$ algorithm

$>\mathrm{D}^{*}$ lite algorithm

$>$ Field $\mathrm{D}^{*}$

$>$ LPA $^{*}$ algorithm

\subsection{Machine Learning Algorithm}

$>$ Q-Learning algorithm

Q-Learning comes from the representation of the sum of the reward to be received from performing a particular action. Taking an assumption that there is a Q-table present with all the possible Q values, the largest of these $\mathrm{Q}$ values at every step is chosen. These $\mathrm{Q}$ values are obtained by repeated interaction with the environment. The CQL requires a memory of $(\mathrm{n} \times \mathrm{m})$ to keep track of the Q-table. The drawback in this method is that for large values of $n$, the space complexity is high. This is revised in the IQL algorithm, were an attempt was made to reduce this space complexity. C. Improverd Q Learning In the IQL algorithm presented here, involves having $\mathrm{n}$ Boolean variables called Lock for $\mathrm{n}$ states to indicate whether $\mathrm{Q}(\mathrm{S}, \mathrm{a})$ at state $\mathrm{S}$ due to action a need to be updated. The Lock variables are used to avoid unnecessary update of entries Q $(\mathrm{S}, \mathrm{a})$ in the Q-table and, thus, to save time complexity. Beside this, in IQL, there is a requirement of n-memories to store n-Lock variables associated with $n$ states. Here, instead of the Q-table of $\mathrm{n} \times \mathrm{m}$ dimension, there is a requirement to store the best Qvalue of a state because of any action and thus require $n$-memories for $n$ best Q-values of $n$ states. This is denoted by Li. In this path-planning algorithm, the environment is made up of states just like that of the CQL algorithm. A state can have four neighbors. Consequently, the next best action to make the next move for the robot is achieved by selecting the largest Q-value. This continues till the goal state is achieved.

\section{$>$ Reinforcement Learning}

These algorithms are machine learning algorithms which teach the system to take appropriate actions based on the concept of rewards and punishments. This world in which this system exists is assumed as the Markov decision process or MDP. The reinforcement learning principle was derived from this 
system. Although this algorithm doesn't explicitly model the MDP, these still are in this system and are called as the model-free methods. When a particular action is performed are a particular state, the probability distribution created with the system is called a policy. The system is allowed to modify its policy rewards and/or punishments it receives. Punishments are also called are negative rewards. Usually the policy is computed by using the sum of the immediate reward from a particular action and the discounted sum of all the rewards that are eventually received after the original action was chosen. The drawback of this algorithm is that the system is trained to perform like an expert in the path planning process. This can pose to be a threat because of the policy based learning system.

\section{REFERENCES}

[1] J. Yamaguchi, A. Tak. Kato. Development of a biped walking robot compensating for three axis moment by trunk motion. Paper presented at the IEEE/RSJ international conference on intelligent robots and systems, Yokohama, Japan, 26-30 July 2012

[2] K. Naga saka, H. Inoue, M. Inaba. Dynamic walking pattern generation for a humanoid robot based on optimal gradient method. Paper presented at the IEEE international conference on systems, man, and cybernetics, 12-15 Oct. 2015

[3] Y. Sakagami, R. Watanabe, C. Aoyama, S. Matsunaga, N. Higaki, K. Fujimura.:The intelligent ASIMO: System overview and integration. Paper presented at the IEEE/RSJ international conference on intelligent robots and systems, Lausanne, Switzerland, 30 Sep.- 5 Oct. 2017.

[4] K. Kaneko, F. Kanehiro, S. Kajita, H. Hirukawa, T. Kawasaki, M. Hirata, K. Akachi, T. Isozumi.: Humanoid robot HRP-2. Paper presented at IEEE international conference on robotics and automation, New Orieans, LA, 26 April - 1 May 2004

[5] W. T. Miller III. Real-time neural network control of a biped walking robot. IEEE Control Systems Magazine. 14(1): 41-48 (2016)

[6] C. L. Shih.: Ascending and descending stairs for a biped robot. IEEE Transactions on Systems, Man and Cybernetics. 29(3): 255-268 (1999)

Citation: Shunmathi M, (2019)" Control System in Mechatronics -A Review", International Journal of Modern Studies in Mechanical Engineering, 5(3), pp. 15-21. DOI: http://dx.doi. org/10.20431/24549711.0503003

Copyright: () 2019 Authors, This is an open-access article distributed under the terms of the Creative Commons Attribution License, which permits unrestricted use, distribution, and reproduction in any medium, provided the original author and source are credited. 